A generalised module for the selective extracellular accumulation of recombinant proteins

Sevastsyanovich et al.

C Biomed Central 


\title{
A generalised module for the selective extracellular accumulation of recombinant proteins
}

\author{
Yanina R Sevastsyanovich ${ }^{1}$, Denisse L Leyton ${ }^{1}$, Timothy J Wells ${ }^{1}$, Catherine A Wardius ${ }^{1}$, Karina Tveen-Jensen ${ }^{1}$, \\ Faye C Morris ${ }^{1}$, Timothy J Knowles ${ }^{2}$, Adam F Cunningham ${ }^{1}$, Jeffrey A Cole ${ }^{3}$ and lan R Henderson ${ }^{1 *}$
}

\begin{abstract}
Background: It is widely believed that laboratory strains of Escherichia coli, including those used for industrial production of proteins, do not secrete proteins to the extracellular milieu.

Results: Here, we report the development of a generalised module, based on an E. coli autotransporter secretion system, for the production of extracellular recombinant proteins. We demonstrate that a wide variety of structurally diverse proteins can be secreted as soluble proteins when linked to the autotransporter module. Yields were comparable to those achieved with other bacterial secretion systems.

Conclusions: The advantage of this module is that it relies on a relatively simple and easily manipulated secretion system, exhibits no apparent limitation to the size of the secreted protein and can deliver proteins to the extracellular environment at levels of purity and yields sufficient for many biotechnological applications.
\end{abstract}

Keywords: Autotransporter, Escherhichia coli, Recombinant protein production, Secretion

\section{Background}

Escherichia coli is the preferred host for recombinant protein production (RPP) in both a research and industrial setting. The popularity of E. coli stems from attributes that include high growth rates in inexpensive media, high product yields, simple process scale-up and safety [1]. The choice of alternative hosts for RPP is predicated on the inability of $E$. coli to achieve adequate production of a target protein. A predominant reason for the selection of an alternative host is the apparent inability of laboratory strains of E. coli to secrete proteins to the extracellular milieu. Targeting recombinant proteins to the culture medium has several advantages over intracellular accumulation of the desired protein including overcoming problems with product toxicity, degradation, aggregation and incorrect folding [1,2]. In principle, it will reduce the number of downstream processing steps due to the ease of product recovery, the reduction in the number and quantity of process impurities and absence of laborious refolding experiments to isolate an active molecule [1].

\footnotetext{
* Correspondence: i.r.henderson@bham.ac.uk

${ }^{1}$ School of Immunity and Infection, University of Birmingham, Edgbaston, Birmingham B15 2TT, United Kingdom

Full list of author information is available at the end of the article
}

Several non-specific strategies for extracellular accumulation of recombinant proteins have been developed for E. coli including genetically or chemically altering strains to promote protein leakage from the periplasmic space to the culture medium $[3,4]$. Unfortunately, this results in large numbers of process impurities in the form of lipids, polysaccharides and proteins derived from the periplasm space and outer membrane $(\mathrm{OM})$. Conversely, if bacterial secretion systems could be manipulated to selectively secrete a desired target protein into the culture medium, in a controlled and predictable manner, it would drastically reduce costs and increase efficiency in bioprocessing [5]. The bacterial type 1, 2, 3 and chaperone-usher systems have been manipulated to secrete foreign proteins from E. coli and other Gramnegative bacteria [6-9]. However, their use for RPP is hampered by the debatable nature of the secretion signals, their molecular complexity (which results in species and/or substrate specificity) and the limited accumulation of the target protein [2]. Extensive genetic manipulation is required to make these systems tractable.

In contrast, the Type 5, or Autotransporter (AT), system has been utilised widely to successfully secrete a variety of heterologous target molecules to the bacterial 
cell surface in a process called Autodisplay [10-14]. ATs are widely distributed among Gram-negative bacteria [15-17]. The precursor protein contains an N-terminal signal sequence, which mediates Sec-dependent protein export into the periplasm, a passenger domain encoding the effector function and a C-terminal domain mediating translocation of the passenger domain across the OM $[16,18,19]$. The effector portion of the molecule displays functional and structural heterogeneity and can be substituted with heterologous proteins $[14,16]$. Whilst successful in delivering a diverse variety of molecules to the cell surface, the AT system has not been successfully adapted for accumulation of heterologous proteins in the culture medium. The system can be engineered to release the heterologous passenger protein into the culture medium with the use of a protease [14], but the use of such proteases is undesirable for production technologies. Here we demonstrate that an AT module can be utilised not only for cell surface display but also for the accumulation of heterologous proteins in the culture medium without the addition of exogenous protease.

\section{Results}

\section{Extracellular accumulation of heterologous proteins}

Other groups have demonstrated the utility of ATs for Autodisplay of heterologous proteins on the bacterial cell surface [14]. In this case the passenger domain remains covalently attached to the $\beta$-barrel translocating subunit. Unlike the ATs used for Autodisplay, cleavage of the passenger domain of the serine protease ATs of the Enterobacteriaceae (SPATEs) from their cognate $\beta$-barrel is effected by nucleophilic attack of $\beta$-barrel residues on a single residue in the $\alpha$-helix [20]. As such, no exogenous protease is required for liberation of the passenger domain from the $\beta$-barrel and in theory the passenger domain can be completely replaced with a target protein. Thus, we hypothesised that the SPATEs could be used to target heterologous proteins to the extracellular milieu rather than the cell surface. To test this hypothesis, initial experiments focused on the E. coli SPATE protein, Pet [21]. When compared to other members of the SPATE family Pet possesses high amino acid sequence identity and structural similarity: the passenger domain consists of a central $\beta$-helical stem decorated with several discursive subdomains and is connected to the characteristic $\beta$-barrel by a short $\alpha$-helical peptide (Additional file 1 : Figure S1). The gene encoding Pet was synthesised de novo (Additional file 2: Figure S2) and cloned into the pASK-IBA33plus or pET22b expression vectors to create plasmid templates onto which the genes encoding heterolgous proteins could be grafted for further experiments.
To test the ability of Pet to mediate secretion of heterologous proteins into the culture medium we chose proteins with distinctive size, structural and functional signatures. These included the secreted portions of (a) Pertactin from Bordetella pertussis, a component of the acellular whooping cough vaccine $(43.9 \mathrm{kDa})$, (b) YapA, a surface protein from Yersinia pestis (105.2 kDa), (c) Pmp17, a polymorphic surface protein from Chlamydophila abortus (40 kDa), (d) SapA, a putative surface protein from Salmonella enterica serovar Typhimurium $(60.7 \mathrm{kDa})$, (e) the red fluorescent protein mCherry, a derivative of Discosoma sp DsRed (26.7 kDa), (f) the predicted secreted esterase Ag85B, a putative Mycobacterium tuberculosis vaccine candidate $(34.6 \mathrm{kDa})$ and $(\mathrm{g})$ ESAT-6 the major diagnostic marker from $M$. tuberculosis (10 kDa) [17,22-25]. DNA encoding the heterologous proteins was synthesized de novo after codon optimization for expression in E. coli (Additional file 2: Figure S2). Previous Autodisplay experiments suggested the $\mathrm{N}$-terminal portion of AT passenger domains were not required for secretion of heterologous proteins to the bacterial cell surface [14]. To verify if this was also the case for proteins released into the culture medium, different parts of the pet gene were replaced in-frame with heterologous DNA to give rise to fusion proteins as shown in Figure 1A. All of the chimeric proteins were secreted into the culture medium as soluble proteins at levels stoichiometrically similar to the wildtype Pet protein (Figure 1B) confirming the $\mathrm{N}$-terminal portions of the passenger domain are not required for secretion. Similarly, the cleaved $\beta$-barrel accumulated in the $\mathrm{OM}$ at levels indistinguishable from wild-type protein (Additional file 3: Figure S3). To authenticate the identities of all secreted proteins, bands were excised from polyacrylamide gels and subjected to mass spectrometry; the appropriate protein was confirmed in each case (Additional file 4: Table S1). Finally, yields of Pertactin and ESAT- 6 from Pet fusion constructs in shake flasks were calculated and accounted for as much as $5 \%$ total cell protein, depending on the expression strain and conditions. For ESAT6-Pet variants and Pertactin-Pet concentrations of 5.4 and $1 \mathrm{mg} / \mathrm{l}$ respectively were achieved after expression in E. coli BL21*.

\section{Heterologous proteins are secreted}

To demonstrate that the presence of heterologous proteins in the culture medium was due to secretion rather than leakage from the periplasm or cell lysis, we examined the cellular location of mCherry and ESAT-6. Recently, we described a mutation in Pet (Pet*) that disrupts the interdomain cleavage site such that the passenger domain is completely translocated to the cell surface but remains covalently attached to the $\beta$-domain [26]. This mutation was introduced into mCherry-Pet-BP 

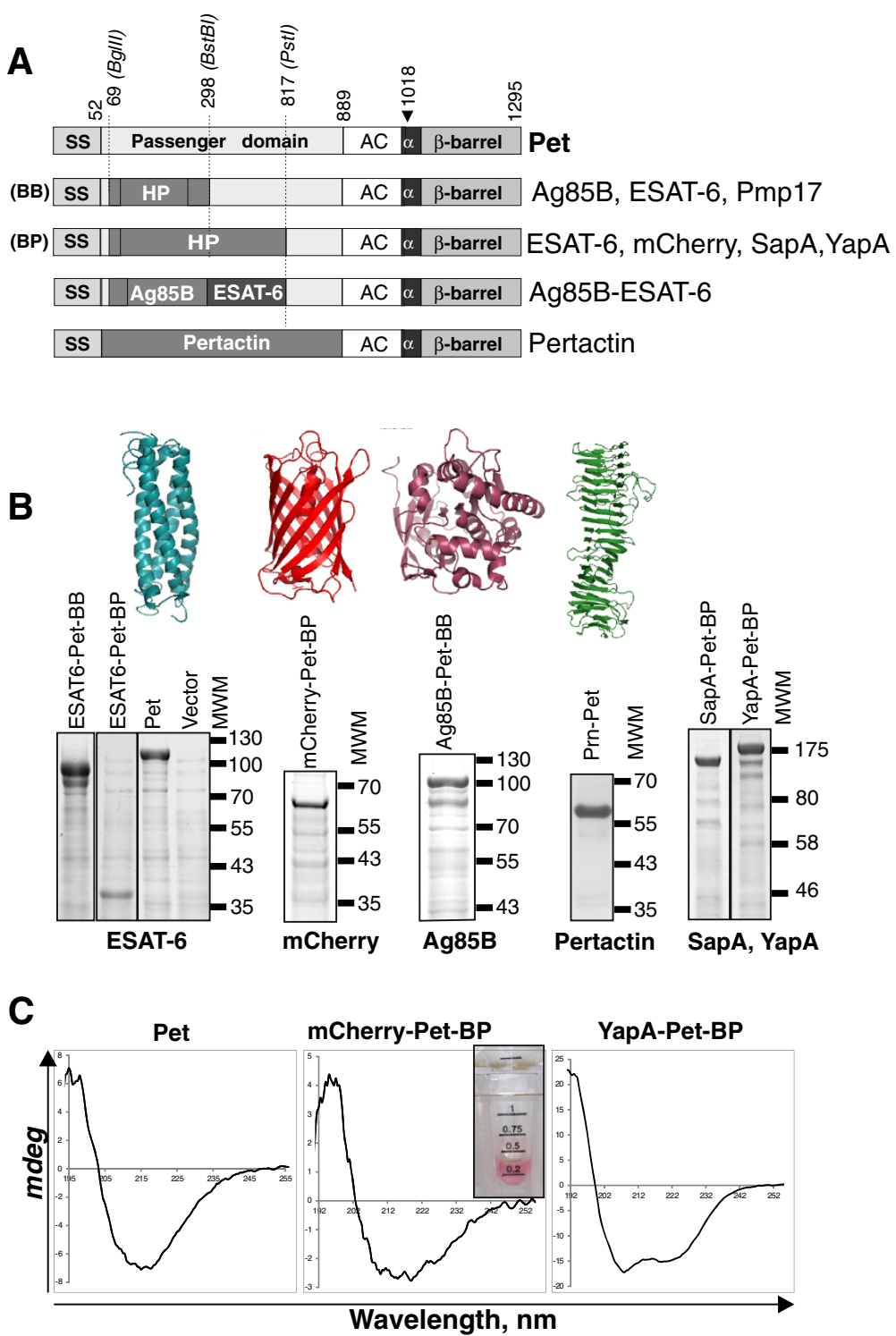

Figure 1 AT-mediated accumulation of heterologous proteins in the culture medium. (A) Schematic diagram of Pet fusion constructs. Heterologous protein insertions in the Pet passenger domain are shown by dark boxes marked HP or with the name of the protein, and are also listed on the right. Abbreviations BB and BP on the left refer to the type of protein fusion generated by insertion of foreign DNA into the pet gene between the restriction sites Bgll and BstBI or Bglll and Pstl, respectively. The co-ordinates above the figure are given for the amino acids derived from the de novo synthesised pet gene. The positions of these sites in the context of the quaternary structure are depicted in Additional file 1: Figure S1. The arrow at position 1018 denotes the cleavage site in the a-helix that effects release of the passenger domain into the culture medium. Modification of this site results in surface display of molecules (Figure 2). The abbreviations SS, AC and a denote the positions of the signal sequence, autochaperone domain and a-helix, respectively. (B) The presence of secreted heterologous proteins in the culture medium was detected by SDS-PAGE or immunoblotting with anti-Pet. Equivalent volumes of medium were analysed. The structures of several heterologous proteins are depicted (not to scale). (C) Investigation of the folded state of secreted heterologous fusion proteins. Far-UV CD spectra of Pet and several heterologous proteins are shown in millidegrees (mdeg). mCherry-Pet-BP harvested from the culture supernatant is shown.

and ESAT6-Pet-BB to create mCherry* and ESAT6*, respectively. In each case no passenger domain accumulates in the culture medium and full length versions can be detected in the OM (Figure 2A and Additional file 5: Figure S4). Immunofluorescence studies of bacteria expressing Pet*, mCherry* and ESAT6* with specific antibodies revealed surface localisation of passenger domains whereas with the native cleavage site there was negligible staining and protein accumulated in the medium (Figure 2B and Figure 1B). Flow cytometry confirmed these observations (Additional file 5: Figure S4). These experiments demonstrate that the 


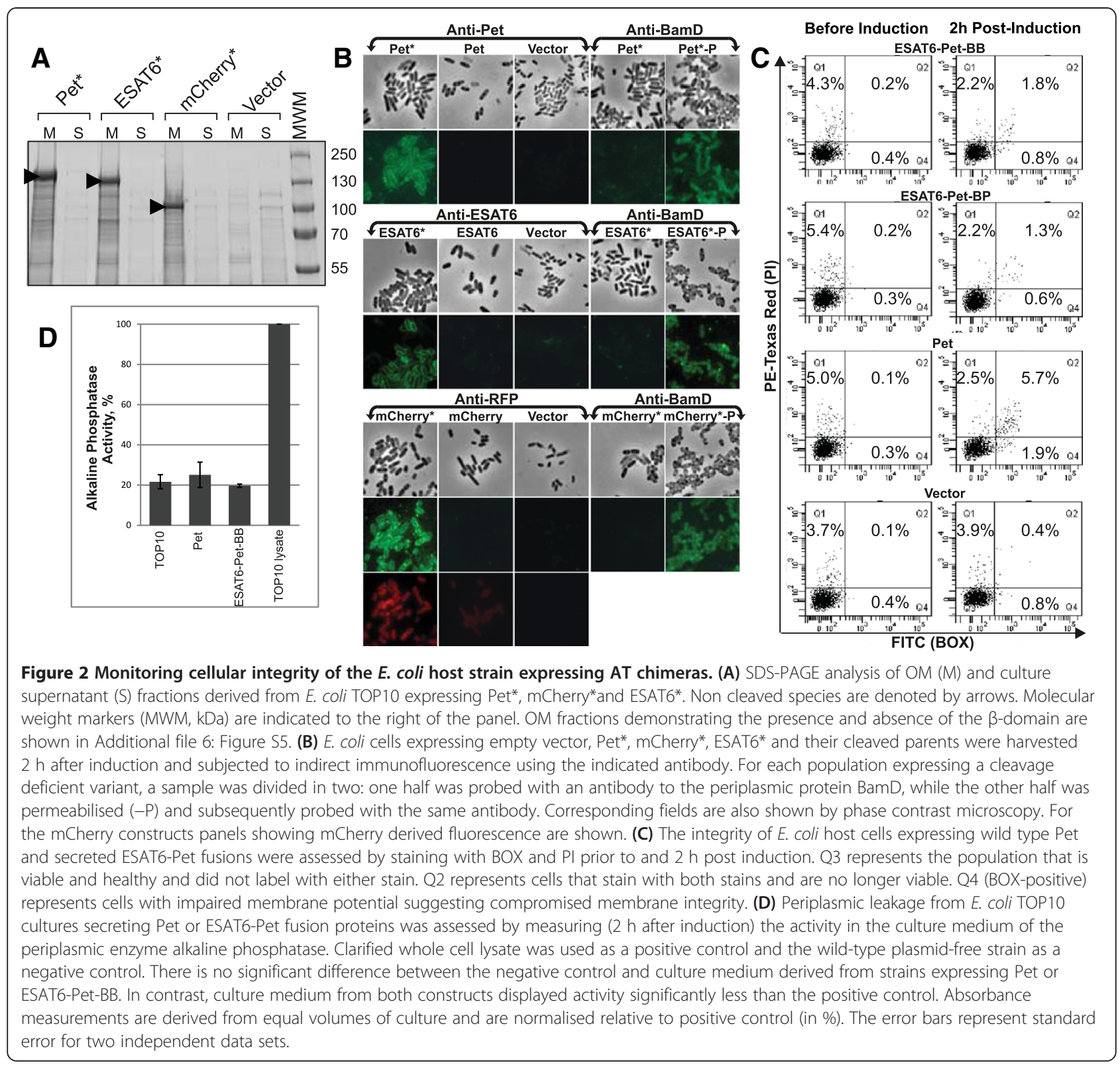

heterologous fusions are expressed and actively translocated to the cell surface before cleavage. Crucially, probing with antibodies directed at the periplasmic protein BamD [27] revealed labelling was not due to ingress of antibodies into the bacterial cell since cells did not label unless permeabilised by chemical treatment (Figure 2B); hence secretion occurred without major loss of membrane integrity. To ensure proteins were not released into the culture medium by cell lysis upon induction of expression, staining with propidium iodide (PI) and Bis-(1,3-dibutylbarbituric acid) trimethine oxonol (BOX) was used to assess cell viability and the integrity of the cell envelope of bacteria secreting heterologous fusions. Importantly, flow cytometry analyses of cultures expressing ESAT6-Pet-BB, ESAT6-Pet-BP and Pet proteins revealed that the majority of cells remain healthy and alive during protein secretion with only negligible increases in the number of $\mathrm{BOX}$ - or $\mathrm{PI}+$ BOX-positive cells after induction of protein expression compared to uninduced cultures (Figure 2C). Finally, assays for alkaline phosphatase, a periplasmic enzyme, demonstrate no leakage of periplasmic proteins after expression of heterologous fusions (Figure 2D). Taken together these data indicate that the presence of secreted proteins in the culture media is not due to cell lysis or periplasmic leakage, but active secretion. Additionally, the presence of ESAT- 6 and fluorescent mCherry on the bacterial cell surface of cultures expressing mCherry* 
and ESAT6*, indicated the Pet AT-module, lacking the cleavage site, can also be used for autodisplay of functional proteins (Figure 2B).

\section{Secreted proteins are soluble, folded and can be modified} To be useful as a method of RPP, the AT system must be able to secrete soluble, folded and functional proteins into the culture medium. To test if the chimeric proteins were natively folded after secretion, we harvested the proteins from the culture supernatant fractions and subjected them to analysis by circular dichroism (CD) (Figure 1C). The structure of YapA is unknown, however bioinformatic analyses predicted YapA to possess a mixed $\alpha$-helical $/ \beta$-strand conformation whereas structural data reveal mCherry adopts a $\beta$-barrel conformation [24]. CD spectra of YapA showed minima at $222 \mathrm{~nm}$ and $208 \mathrm{~nm}$ and maxima at $195 \mathrm{~nm}$ indicative of a folded protein with mixed $\alpha$-helical $/ \beta$-strand content. Consistent with their natively folded $\beta$-strand conformations, CD spectra for Pet and mCherry show minima at $218 \mathrm{~nm}$ and maxima at $195 \mathrm{~nm}$. Additionally, mCherry purified from the culture supernatant fraction shows fluorescence indicating a folded protein with functional activity (Figure 1C).

Having established the Pet AT system can support the specific secretion of some heterologous proteins in a folded and functional state, we investigated whether the system could secrete modified chimeras consisting of proteins with disulphide bonded cysteine residues, purification tags or multiprotein complexes. Proteins targeted for secretion by the AT mechanism have to traverse the periplasm, a highly oxidising cellular compartment where formation of disulphide bonds between cysteine residues is catalysed by DsbA [28]. Pmp17-Pet-BB, possessing 7 cysteines, was expressed in E. coli TOP10 $d s b A$ and accumulated in the culture medium at levels consistent with wild-type Pet (Figure 3A). No full-length protein could be detected in wild-type E. coli TOP10. However, degradation products were observed in the extracellular milieu. These products are consistent with a degraded passenger domain lacking the ca. $24 \mathrm{kDa} \mathrm{N}$-terminal region containing the cysteines and based on previous experiments is the result of the action of the periplasmic protease DegP [26,29]. When the $\mathrm{N}$-terminal region containing the cysteines is removed by DegP-mediated degradation the disulphide bonds cannot form and secretion is no longer stalled. These results are consistent with observations made for Pet and other ATs that demonstrate disulphide bond formation and partial folding of native or heterologous polypeptides hinders their secretion $[26,29,30]$. Fusion tags might be desirable for downstream purification applications. N-terminal addition of a $\mathrm{His}_{6}$-tag to the SapA and Pmp17 passenger domains, as well as on wild-type and truncated Pet derivatives, did not interfere with secretion of target proteins to the extracellular milieu (Figure 3A). Previously, we demonstrated the system is capable of secreting proteins with HA- and FLAG-tags [26]. Notably, the multicomponent chimera containing both Ag85B and ESAT-6 proteins could also be secreted by Pet (Figure 3A). To determine whether the AT module can be utilised for production of heterologous proteins in other Gram-negative bacteria we investigated expression of ESAT6-Pet-BP and ESAT6-Pet $\Delta * 6$ (see below) in S. Typhimurium SL1344 and its avirulent
A

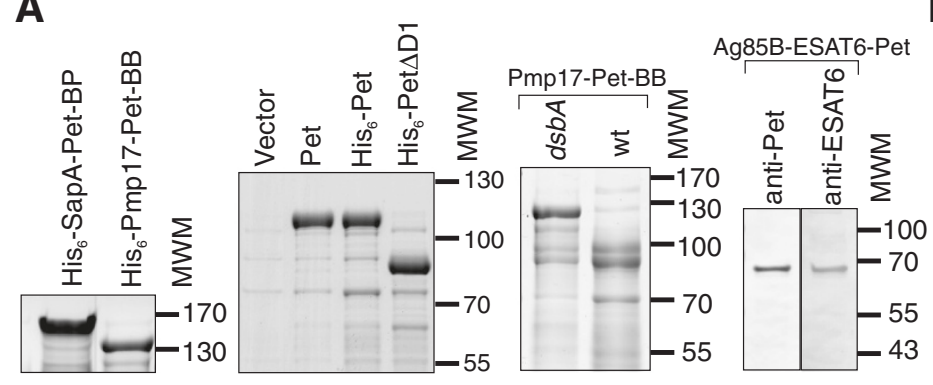

B

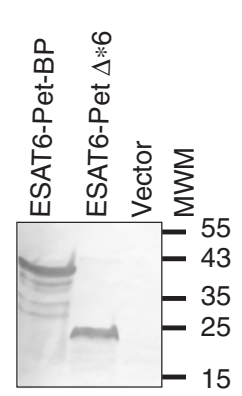

Figure 3 Modification of the Pet-AT secretion platform. (A) SapA, Pmp17, Pet and a Pet derivative (Pet $\Delta$ D1) lacking the serine protease domain were modified to add a His $\sigma_{6}$ Tag to the $\mathrm{N}$-terminus of the secreted passenger domain. The His-tagged Pmp17 protein was expressed in an E. coli TOP10 dsbA strain and the rest of proteins were produced in the wild-type E. coli TOP10. In all cases the proteins are well secreted. The cysteine-containing Pmp17 was expressed in E. coli TOP10 (wt) and a dsbA- derivative. A full length protein is present in the E. coli TOP10 dsbA derivative but not the $E$. coli TOP10 parent strain. Break down products are apparent and correspond to proteins with a truncated $\mathrm{N}$-terminus. A multicomponent construct was created by fusing DNA encoding Ag85 to ESAT-6 and Pet (see Figure 1A) to encode a single polypeptide chain contiguous with the AT-translocation unit. This latter chimera was detected in the culture supernatant with antibodies directed at Pet and ESAT-6. Equivalent amounts of culture supernatant fractions were analysed by SDS-PAGE. (B) Secretion of heterologous fusions from S. Typhimurium. Culture medium from S. enterica SL1344 strains expressing ESAT6-Pet-BP and ESAT6-Pet $\Delta^{*} 6$ (see Figure 4) were harvested and analysed by SDSPAGE and detected by immunoblotting with a polyclonal antibody to ESAT-6. In all SDS-PAGE gels the positions of the molecular weight markers $(\mathrm{MWM}, \mathrm{kDa}$ ) are depicted at the right side of the panel. The equivalent OM fractions demonstrating the presence of the cleaved $\beta$-barrel are shown in Additional file 5: Figure S4. 
derivative SL3261. In both cases, ESAT-6 was secreted into the culture medium at levels similar to that for Pet (Figure 3B). These data demonstrate the versatility of the Pet AT-module for secreting proteins and multiprotein complexes into the culture medium.

\section{Determination of the minimal construct mediating secretion}

The constructs described above possessed at least 100 amino acids upstream of the predicted Pet $\beta$-barrel. This stretch of amino acids encompasses the $\alpha$-helical peptide spanning the $\beta$-barrel lumen and the Autochaperone (AC) domain. Previously, the AC domain has been implicated in secretion of passenger domains, where contemporaneous folding of the $\beta$-helix and a Brownian ratchet mechanism provide the vectorial impetus for secretion $[31,32]$. Here we sought to determine the precise length of the minimal functional translocation domain for Pet and determine whether the AC-domain is required for secretion of heterologous proteins to the growth medium. To this end, we examined secretion of ESAT-6-Pet-BP and 20 derivatives harbouring sequential truncations (Figure 4 and Additional file 6: Figure S5). All Pet derivatives, including the smallest variant tested that contains only the predicted $\alpha$-helix and the downstream $\beta$-barrel domain, could sustain ESAT-6 secretion into the culture medium (Figure 4). ESAT-6 secreted by this Pet fragment contains only the 9 amino acids of the wild-type Pet passenger domain $\alpha$-helix that are adjacent to the cleavage site. Two recent reports implicated a conserved tryptophan residue (W985) in the AC domain in secretion of some SPATEs [33,34]. Interestingly, ESAT6-Pet $\Delta * 17$ to $\Delta^{*} 20$ proteins lack the predicted Pet AC domain altogether but are well secreted (Figure 4). To further test a role for W985 in secretion, this amino acid and three other conserved and closely positioned residues (I983, L987 and G989) were mutated to Ala and Lys in the secretion-competent ESAT6-Pet $\Delta * 6$. All mutated proteins were secreted into the growth medium as efficiently as the ESAT6-Pet $\Delta * 6$ and retained a cleaved $\beta$-domain in the OM (Additional file 7: Figure S6). These data further support the view that the AC domain is not required for secretion per se but is essential for folding of native AT polypeptides.

Next we investigated whether other SPATE proteins could support secretion of heterologous proteins in a manner analogous to Pet. Pic [35] belongs to a clade of the SPATEs that is evolutionarily distinct from that harbouring Pet. Alignment of Pet and Pic protein sequences from the beginning of the predicted AC domains shows $68 \%$ identity and $80 \%$ similarity (Additional file 8 : Figure S7). Based on the alignment we constructed ESAT6-Pic fusions containing C-terminal Pic fragments
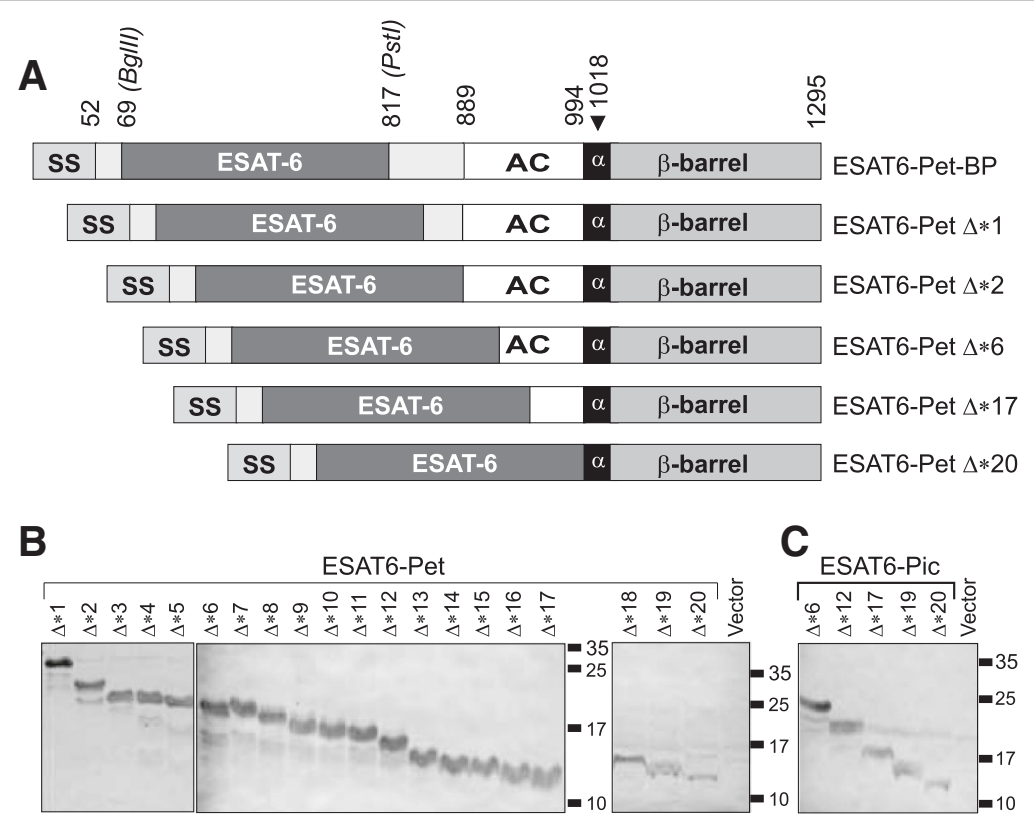

Figure 4 Identification of the minimal AT module permitting secretion of heterologous proteins to the culture supernatant fraction. (A) Schematic of ESAT6-Pet-BP protein fusion and some truncations created to determine the minimal C-terminal Pet fragment capable of ESAT-6 secretion. The $\Delta^{*} 1, \Delta^{*} 2, \Delta^{*} 6, \Delta^{*} 17$ and $\Delta^{*} 20$ Pet truncations are shown while for simplicity the intermediate variants $\Delta^{*} 3-\Delta^{*} 5, \Delta^{*} 7-\Delta^{*} 16, \Delta^{*} 18$ and $\Delta^{*} 19$ are omitted. Abbreviations are the same as in Figure 1. (B and C) Detection by western immunoblotting of ESAT6-Pet (B) and ESAT6Pic (C) chimeras expressed in E. coli TOP10. The TCA-precipitated culture supernatants were analysed by SDS-PAGE and probed with polyclonal anti-ESAT6. The equivalent OM fractions demonstrating the presence of the cleaved $\beta$-barrel in the OM are shown in Additional file 6: Figure S5. 
equivalent to those for ESAT6-Pet variants. All ESAT6Pic fusions were efficiently secreted (Figure 4C) allowing us to conclude that $\beta$-barrels from SPATE proteins other than Pet can be used to secrete proteins to the culture medium.

\section{Discussion}

E. coli was the first organism to be used for industrial scale production of recombinant proteins. Since then, a large number of proteins produced in $E$. coli have successfully reached the market including human interferons, interleukins, and granulocyte stimulating factor. However, the dominance of $E$. coli in industrial bioprocessing is waning with only ca. $40 \%$ of new recombinant protein pharmaceuticals being produced using prokaryotic cells. The diminution of $E$. coli is strongly related to that fact that strains of $E$. coli used for industrial scale protein production do not effectively secrete proteins into the extracellular environment. Solutions to this problem would effectively reposition E. coli as the host of choice for industrial recombinant protein production. To achieve selective accumulation of recombinant proteins in the extracellular milieu, a Gram-negative protein secretion system must be harnessed. While secretion of heterologous proteins through the Type 1-3 systems has been achieved, the complexity of these systems limits the nature and size of the proteins that can be targeted for secretion. The work described here demonstrates that an AT module, based on SPATE proteins, can be used for the targeted secretion of chimeric proteins into the culture media of Gram-negative bacteria. Importantly, we have demonstrated that this platform may be used for the specific accumulation of folded heterologous proteins with functional, structural and size heterogeneity and for multicomponent complexes (Figure 1). The ability to selectively accumulate target proteins in the culture medium, away from the majority of the process impurities associated with expression in other compartments, makes this system attractive for adoption in industrial RPP applications since extracellular expression of proteins in a folded active form enables simplification of the purification process and significantly reduces downstream processing. However, effective utilisation of the AT module for generalised RPP necessitates achieving yields of target proteins at industrial scale and at concentrations competitive with alternative technologies. Importantly, the yields achieved here are consistent with levels achieved for other E. coli protein secretion systems [1,36]. However, in the experiments conducted here, only 50\% of the expressed protein is targeted to the extracellular milieu, the remainder accumulating at the outer membrane. Furthermore, these yields were obtained in small-scale non-optimised conditions. Thus, an optimised secretion platform, in a controlled optimised fermentation process, would be expected to generate higher protein yields.

Live attenuated bacterial vectors offer the opportunity to deliver vaccine candidates inside human cells thereby eliciting a protective immune response against both infectious and non-infectious disease e.g. tumours. While the induction of antibody has been demonstrated in many animal models, the anticipated induction of cell-mediated immunity has been disappointing [37]. There are several reasons for this including (1) inefficient production of the recombinant heterologous antigen and (2) after invading professional phagocytic cells the live vector remains in the phagosome such that its antigens do not reach the cytosolic processing pathway. Surface display of AT-fusions in live-attenuated vaccine strains offers potential to overcome these problems [14]. Here we have demonstrated that the AT-module can be used in conjunction with liveattenuated Salmonella strains and demonstrated the successful surface display of folded functional heterologous proteins (Figure 2 and 3). Unfortunately, previous experiments using surface display have also failed to induce substantial protective immunity [14]. However, the ability of live attenuated vectors to secrete antigens intracellularly may enhance presentation of the antigens to the immune system and provide the desired protective response [38]. Thus, our demonstrated expression of secreted heterologous protein constructs in live attenuated Salmonella strains offers the possibility of developing a platform for the delivery of multivalent vaccines based on the continuous secretion of proteins in vivo in which significant cell mediated immunity is generated.

Finally, this work reveals novel insight into the biology of the AT secretion system. Several articles have described the importance of specific amino acid residues for the secretion of passenger domain proteins notably residues present in the AC domain [33,34]. Indeed, recent investigations of BrkA suggested that during secretion portions of the $\mathrm{AC}$ domain are sequestered by a specific domain within the $\beta$-barrel which initiates translocation of the passenger domain to the external environment [39]. Work provided here clearly demonstrates that the AC domain is not essential for secretion since the smallest secretioncompetent constructs completely lack this domain and mutations within the domain do not affect secretion levels (Figure 4 and Additional file 8: Figure S7). These investigations reinforce the concept that the $\mathrm{AC}$ domain is required specifically for folding of the $\beta$-helical passenger domain, although folding of the passenger domain may enhance the rate at which secretion occurs [30].

\section{Conclusions}

In conclusion, we have developed a versatile platform, based on an AT module, which can be used for 
secretion of heterologous fusion proteins to the culture medium in a soluble folded form. Additionally, this system can be used for surface display of heterologous fusions on the bacterial cell surface.

\section{Methods}

Bacterial strains and growth conditions

E. coli strains TOP10 (Invitrogen), NEB $5 \alpha \mathrm{F}^{\mathrm{q}} \mathrm{I}^{\mathrm{a}}$ and JM110 (NEB) were used for cloning. E. coli TOP10, TOP10 dsbA [26] and BL21* (Novagen) and S. enterica SL1344 and SL3261 strains [40] were used for protein expression and secretion. Bacteria were grown at $37^{\circ} \mathrm{C}$ in Luria-Bertani liquid or solid media supplemented with carbenicillin (100 and $80 \mu \mathrm{g} / \mathrm{ml}$, respectively) or kanamycin $(50 \mu \mathrm{g} / \mathrm{ml})$ when appropriate. Protein expression was induced by adding anhydrotetracycline $(200 \mu \mathrm{g} / \mathrm{l})$ or IPTG $(0.5 \mathrm{mM})$ as appropriate and the cultures were incubated for a further $1.5-2 \mathrm{~h}$.

\section{General molecular biology techniques and plasmid construction}

Recombinant DNA manipulations were described elsewhere [41]. Phusion High-Fidelity DNA polymerase (Finnzymes), DNA modifying enzymes, plasmid miniprep and PCR/gel extraction kits (Fermentas, Qiagen and NEB) were used according to the manufacturer's instructions. Oligonucleotides were synthesized by Alta Bioscience and Eurogentec. Sequencing, mass spectrometry, flow cytometry and gel imaging/densitometry were done using the University of Birmingham Functional genomics facility. Codon optimization for the most commonly used codons and de novo synthesis of DNA was done by GenScript, GenArt or Epoch Life Science. Alignments were generated with ClustalX [42] and phylogenetic trees generated with Geneious software (http://www.geneious.com/).

Plasmids used in this study are listed in Additional file 9: Table S2. Primers used for PCR are listed in Additional file 10: Table S3. To construct pASK-Pet, the pet gene was PCR-amplified from pBAD-Pet with BsaI-pet$\mathrm{F}$ and HindIII-pet-R primers and cloned between BsaI/ HindIII sites in pASK-IBA33plus (IBA BioTAGnology). pET-Pet was constructed by cloning the pet gene into the NdeI-HindIII sites of pET22b. pASK-His 6 -Pet and pASK-His ${ }_{6}$-Pet- $\triangle \mathrm{D} 1$ were constructed by replacing the SacI-BglII or SacI-BstBI pet fragment in pASK-Pet with an amplicon generated by PCR with SacI-pet-F and PetSS-BglII-AflII-BstBI-R primers, the latter encoding a $\mathrm{His}_{6}$-tag sequence. To construct pet chimeras the heterologous genes were amplified by PCR using appropriate primer pairs and relevant DNA templates listed in Additional file 2: Figure S2. The PCR-amplified heterologous genes were cloned between BglII/BstBI and BglII/PstI sites in the pet gene in pASK-Pet, pASK-Pet* or pET-Pet. pASK-Ag85B-ESAT6-Pet was constructed by inserting PCR-amplified esxA gene (ESAT-6) between BstBI-PstI sites in pASK-Ag85B-Pet-BB. Constructs pASK-ESAT6-Pet $\Delta^{*} 1$ to $\Delta^{*} 20$ were made by replacing the PstI-HindIII fragment in pASK-ESAT6Pet-BP with the shorter pet gene fragments generated by PCR with one of the forward primers (PstI-TSYQdel1-F to PstI-YKAF-del20-F) and HindIII-pet-R as a reverse primer. Equivalent constructs encoding ESAT6-Pic chimeras were generated by replacing the PstI-HindIII pet fragment in pASK-ESAT6-Pet-BP with the pic fragment amplified from pPic1 [35] using one of the forward primers SbfI-FKAG-Pic-del6-F to SbfIYKNF-Pic-del20-F) and HindIII-Pic-end-R as a reverse primer. Codons encoding 1974, W985, L987 and G989 were mutated by site directed mutagenesis using primers BglII-ESAT6-F and HindIII-pet-R as previously described [43]. All constructs generated in this study were sequenced to confirm the veracity of the nucleotide modifications.

\section{Preparation and analysis of proteins}

Proteins were visualised by Coomassie staining after SDS-PAGE on standard [44] or precast (Precise 4-20\% Tris/HEPES, ThermoFisher; NuPAGE 4-12\% Bis-Tris/ MES, Invitrogen) polyacrylamide protein gels or by western immunoblotting as previously described [45]. Rabbit polyclonal antibodies against Pet passenger domain (1:5000 dilution) [46], ESAT-6 (Abcam; 1:2000 dilution) and mCherry (anti-RFP, Abcam; 1:2000) were used for western immunoblotting. Secondary alkaline phosphatase-conjugated goat anti-rabbit antibodies and NBT/BCIP (Nitro blue tetrazolium chloride/5Bromo-4-chloro-3-indolylphosphate) substrate were purchased from Sigma. Protein concentrations were determined spectrophotometrically and by SDS-PAGE comparisons with known quantities of purified protein: Bovine serum albumin, Ovalbumin and Lysozyme (Sigma).

Cellular fractions were prepared essentially as described previously [44]. His ${ }_{6}$-tagged proteins were purified by affinity chromatography on Ni-agarose following manufacturer's instructions (WebScientific). Briefly, $400 \mathrm{ml}$ cultures were grown and protein expression was induced as described above. Culture supernatants were harvested and sterilised as above, supplemented with $1 \mathrm{mM}$ PMSF and then concentrated through Vivaspin centrifugation device (Sartorius). The concentrated supernatant fractions were passed over a Ni-agarose affinity chromatography column under native conditions using $50 \mathrm{mM}$ sodium phosphate, $500 \mathrm{mM}$ $\mathrm{NaCl}, 10 \mathrm{mM}$ imidazole (pH7.5) as binding buffer and $50 \mathrm{mM}$ sodium phosphate, $500 \mathrm{mM} \mathrm{NaCl}, 500 \mathrm{mM}$ imidazole (pH7.5) as elution buffer. 
To test viability, bacterial cells $\left(\sim 10^{5}-10^{6}\right.$ cells $\left./ \mathrm{ml}\right)$ were diluted in $1 \mathrm{ml}$ filter-sterilised Dulbecco's PBS supplemented with $10 \mu \mathrm{l}$ of working solutions of PI and BOX (5 and $10 \mu \mathrm{g} / \mathrm{ml}$ respectively; Sigma) and analysed immediately on FACSAria II (BD Biosciences) using $488 \mathrm{~nm}$ laser [47]. Side and forward scatter data and fluorescence data from $10^{4}$ particles were collected. Optical filters used to measure green and red fluorescence were 502LP, 530/30BP (FITC) and 610LP, 616/23BP (PETexas Red), respectively. To analyse surface localisation of proteins by indirect flow cytometry, cells were washed in PBS and incubated at RT with 1\% BSA in PBS. Cells were then incubated for $1 \mathrm{~h}$ at RT with primary antibody diluted in PBS (anti-Pet, 1:500; anti-ESAT6, 1:500; anti-mCherry, 1:800) followed by 3 PBS washes and final incubation with Alexa Fluor ${ }^{\circledR} 488$ goat anti-rabbit IgG (1:500; Invitrogen). Cells were washed as before and analysed on a FACSAria II as above.

Proteins in live or fixed bacterial cells were detected by indirect Immunofluorescence as previously described [26]. Cells were visualized using either phase contrast or fluorescence using a Leica DMRE fluorescence microscope-DC200 digital camera system. Exposure time was $118 \mathrm{~ms}$. The Garen and Levinthal [48] assay of Alkaline Phosphatase activity was used based on conversion of $p$-nitrophenylphosphate ( $\mathrm{pNPP}$ ) substrate into yellow product with absorbance at $410 \mathrm{~nm}$. Far-UV CD measurements from 190 to $260 \mathrm{~nm}$ were collected on a JASCO J-715 spectropolarimeter at room temperature, as described previously [26]. Protein structures were modelled in Swiss-Model [49] or Phyre [50] and were visualised using PyMol (http: \www. pymol.org). Secondary structures were predicted with PsiPred [51].

\section{Additional files}

Additional file 1: Figure S1. Model of the Pet structure.

Additional file 2: Figure S2. Nucleotide sequences of the de novo synthesised pet gene and heterologous DNA encoding proteins targeted for secretion.

Additional file 3: Figure S3. AT-mediated accumulation of heterologous proteins in the culture medium

Additional file 4: Table S1. Mass spectrometry analysis of some recombinant protein fusions with Pet.

Additional file 5: Figure S4. Surface localisation of non cleaved Pet and fusion proteins.

Additional file 6: Figure S5. Identification of minimal AT module permitting secretion of heterologous proteins to the culture supernatant fraction.

Additional file 7: Figure S6. Impact of conserved amino acids from the AC domain on secretion of heterologous proteins.

Additional file 8: Figure S7. Comparison of the Pic and Pet SPATE proteins. Additional file 9: Table S2. Plasmids used in this study.

Additional file 10: Table S3. Primers used in this study.

\section{Abbreviations}

RPP: recombinant protein production; AT: Autotransporter; OM: Outer Membrane; SPATEs: Serine Protease Autotransporter of the Enterobacteriaceae; AC: Autochaperone; BOX: Bis-(1,3-dibutylbarbituric acid) trimethine oxonol; PI: Propidium lodide.

\section{Competing interests}

The authors declare that they have no competing interests. The work described in this article has been submitted for patent protection by the University of Birmingham.

\section{Author contributions}

$\mathrm{IRH}, \mathrm{JAC}$ and AFC designed the project. DLL, YRS and KT designed and constructed the expression vectors. YRS, DLL and TJK purified proteins and performed CD analyses. YRS, TJW, FCM and CAW prepared and analysed bacterial fractions. TJW performed the immunofluorescence studies. YRS performed the alkaline phosphatase experiments and flow cytometry analyses. All authors contributed to the preparation of the manuscript. All authors read and approved the final manuscript.

\section{Acknowledgements}

This work was supported by grants from BBSRC to IRH and MRC to IRH, AFC and JAC. We thank Dr Lewis E. H. Bingle (University of Sunderland) for critical reading of the manuscript and Dr Raul Pacheco-Gomez for advice on CD.

\section{Author details}

${ }^{1}$ School of Immunity and Infection, University of Birmingham, Edgbaston, Birmingham B15 2TT, United Kingdom. ${ }^{2}$ School of Cancer Sciences,

University of Birmingham, Edgbaston, Birmingham B15 2TT, United Kingdom. ${ }^{3}$ School of Biosciences, University of Birmingham, Edgbaston, Birmingham B15 2TT, United Kingdom.

Received: 8 April 2012 Accepted: 11 May 2012

Published: 28 May 2012

\section{References}

1. Mergulhao FJ, Summers DK, Monteiro GA: Recombinant protein secretion in Escherichia coli. Biotechnol Adv 2005, 23:177-202.

2. Sandkvist M, Bagdasarian M: Secretion of recombinant proteins by Gramnegative bacteria. Curr Opin Biotechnol 1996, 7:505-511.

3. Shokri A, Sanden AM, Larsson G: Cell and process design for targeting of recombinant protein into the culture medium of Escherichia coli. Appl Microbiol Biotechnol 2003, 60:654-664

4. Choi JH, Lee SY: Secretory and extracellular production of recombinant proteins using Escherichia coli. Appl Microbiol Biotechnol 2004, 64:625-635.

5. Henderson IR, Navarro-Garcia F, Nataro JP: The great escape: structure and function of the autotransporter proteins. Trends Microbiol 1998, 6:370-378.

6. Chen $\mathrm{H}$, Schifferli DM: Comparison of a fimbrial versus an autotransporter display system for viral epitopes on an attenuated Salmonella vaccine vector. Vaccine 2007, 25:1626-1633.

7. Tzschaschel BD, Guzman CA, Timmis KN, de Lorenzo V: An Escherichia coli hemolysin transport system-based vector for the export of polypeptides: export of Shiga-like toxin IleB subunit by Salmonella typhimurium aroA. Nat Biotechnol 1996, 14:765-769.

8. Widmaier DM, Tullman-Ercek D, Mirsky EA, Hill R, Govindarajan S, Minshull J, Voigt CA: Engineering the Salmonella type III secretion system to export spider silk monomers. Mol Syst Biol 2009, 5:309.

9. Majander K, Anton L, Antikainen J, Lang H, Brummer M, Korhonen TK, Westerlund-Wikstrom B: Extracellular secretion of polypeptides using a modified Escherichia coli flagellar secretion apparatus. Nat Biotechnol 2005, 23:475-481.

10. Ruiz-Olvera P, Ruiz-Perez F, Sepulveda NV, Santiago-Machuca A, MaldonadoRodriguez R, Garcia-Elorriaga G, Gonzalez-Bonilla C: Display and release of the Plasmodium falciparum circumsporozoite protein using the autotransporter MisL of Salmonella enterica. Plasmid 2003, 50:12-27.

11. Luria-Perez R, Cedillo-Barron L, Santos-Argumedo L, Ortiz-Navarrete VF, Ocana-Mondragon A, Gonzalez-Bonilla CR: A fusogenic peptide expressed on the surface of Salmonella enterica elicits CTL responses to a dengue virus epitope. Vaccine 2007, 25:5071-5085. 
12. Kjaergaard K, Hasman H, Schembri MA, Klemm P: Antigen 43-mediated autotransporter display, a versatile bacterial cell surface presentation system. J Bacteriol 2002, 184:4197-4204.

13. Klauser T, Pohlner J, Meyer TF: Extracellular transport of cholera toxin B subunit using Neisseria IgA protease beta-domain: conformationdependent outer membrane translocation. EMBO J 1990, 9:1991-1999.

14. Jose J, Meyer TF: The autodisplay story, from discovery to biotechnical and biomedical applications. Microbiology and molecular biology reviews: MMBR 2007, 71:600-619.

15. Desvaux M, Khan A, Beatson SA, Scott-Tucker A, Henderson IR: Protein secretion systems in Fusobacterium nucleatum: genomic identification of Type 4 piliation and complete Type $V$ pathways brings new insight into mechanisms of pathogenesis. Biochim Biophys Acta 2005, 1713:92-112.

16. Henderson IR, Navarro-Garcia F, Desvaux M, Fernandez RC, Ala'Aldeen D: Type $\mathrm{V}$ protein secretion pathway: the autotransporter story. Microbiology and molecular biology reviews: MMBR 2004, 68:692-744.

17. Henderson IR, Lam AC: Polymorphic proteins of Chlamydia spp.autotransporters beyond the Proteobacteria. Trends Microbiol 2001, 9:573-578.

18. Desvaux M, Parham NJ, Henderson IR: Type V protein secretion: simplicity gone awry? Current issues in molecular biology 2004, 6:111-124.

19. Desvaux M, Parham NJ, Scott-Tucker A, Henderson IR: The general secretory pathway: a general misnomer? Trends Microbiol 2004, 12:306-309.

20. Tajima N, Kawai F, Park SY, Tame JR: A novel intein-like autoproteolytic mechanism in autotransporter proteins. J Mol Biol 2010, 402:645-656.

21. Chaudhuri RR, Sebaihia M, Hobman JL, Webber MA, Leyton DL, Goldberg MD, Cunningham AF, Scott-Tucker A, Ferguson PR, Thomas CM, et al: Complete genome sequence and comparative metabolic profiling of the prototypical enteroaggregative Escherichia coli strain 042. PLoS One 2010, 5:e8801.

22. Andersen $P$, Andersen $A B$, Sorensen $A L$, Nagai $S$ : Recall of long-lived immunity to Mycobacterium tuberculosis infection in mice. J Immunol 1995, 154:3359-3372.

23. Leininger E, Roberts M, Kenimer JG, Charles IG, Fairweather N, Novotny $P$, Brennan MJ: Pertactin, an Arg-Gly-Asp-containing Bordetella pertussis surface protein that promotes adherence of mammalian cells. Proc Natl Acad Sci U S A 1991, 88:345-349.

24. Shaner NC, Campbell RE, Steinbach PA, Giepmans BN, Palmer AE, Tsien RY: Improved monomeric red, orange and yellow fluorescent proteins derived from Discosoma sp. red fluorescent protein. Nat Biotechnol 2004, 22:1567-1572.

25. Yen YT, Karkal A, Bhattacharya M, Fernandez RC, Stathopoulos C Identification and characterization of autotransporter proteins of Yersinia pestis KIM. Mol Membr Biol 2007, 24:28-40.

26. Leyton DL, Sevastsyanovich YR, Browning DF, Rossiter AE, Wells TJ, Fitzpatrick RE, Overduin M, Cunningham AF, Henderson IR: Size and conformation limits to secretion of disulfide-bonded loops in autotransporter proteins. J Biol Chem 2011, 286:42283-42291.

27. Rossiter AE, Leyton DL, Tveen-Jensen K, Browning DF, Sevastsyanovich $Y$, Knowles TJ, Nichols KB, Cunningham AF, Overduin M, Schembri MA, Henderson IR: The essential beta-barrel assembly machinery complex components BamD and BamA are required for autotransporter biogenesis. J Bacterio/ 2011, 193:4250-4253.

28. Gleiter S, Bardwell JC: Disulfide bond isomerization in prokaryotes. Biochim Biophys Acta 2008, 1783:530-534.

29. Jong WS, ten Hagen-Jongman CM, den Blaauwen T, Slotboom DJ, Tame JR, Wickstrom D, de Gier JW, Otto BR, Luirink J: Limited tolerance towards folded elements during secretion of the autotransporter $\mathrm{Hbp}$. Mol Microbiol 2007, 63:1524-1536.

30. Leyton DL, Rossiter AE, Henderson IR: From self sufficiency to dependence: mechanisms and factors important for autotransporter biogenesis. Nat Rev Microbiol 2012, 10:213-225.

31. Oliver DC, Huang G, Nodel E, Pleasance S, Fernandez RC: A conserved region within the Bordetella pertussis autotransporter BrkA is necessary for folding of its passenger domain. Mol Microbiol 2003, 47:1367-1383.

32. Renn JP, Clark PL: A conserved stable core structure in the passenger domain beta-helix of autotransporter virulence proteins. Biopolymers 2008, 89:420-427.

33. Peterson JH, Tian P, leva R, Dautin N, Bernstein HD: Secretion of a bacterial virulence factor is driven by the folding of a C-terminal segment. Proc Natl Acad Sci U S A 2010, 107:17739-17744.
34. Soprova Z, Sauri A, van Ulsen P, Tame JR, den Blaauwen T, Jong WS, Luirink $\mathrm{J}$ : A conserved aromatic residue in the autochaperone domain of the autotransporter $\mathrm{Hbp}$ is critical for initiation of outer membrane translocation. J Biol Chem 2010, 285:38224-38233.

35. Harrington SM, Sheikh J, Henderson IR, Ruiz-Perez F, Cohen PS, Nataro JP: The Pic protease of enteroaggregative Escherichia coli promotes intestinal colonization and growth in the presence of mucin. Infect Immun 2009, 77:2465-2473.

36. Ni Y, Chen R: Extracellular recombinant protein production from Escherichia coli. Biotechnol Lett 2009, 31:1661-1670.

37. Cheminay C, Hensel M: Rational design of Salmonella recombinant vaccines. International journal of medical microbiology: IJMM 2008, 298:87-98.

38. Moingeon P, de Taisne C, Almond J: Delivery technologies for human vaccines. British medical bulletin 2002, 62:29-44.

39. Zhao L, Nguyen NT, Fernandez RC, Murphy ME: Crystallographic characterization of the passenger domain of the Bordetella autotransporter BrkA. Acta crystallographica Section F, Structural biology and crystallization communications 2009, 65:608-611.

40. Gil-Cruz C, Bobat S, Marshall JL, Kingsley RA, Ross EA, Henderson IR, Leyton $D L$, Coughlan RE, Khan M, Jensen KT, et al: The porin OmpD from nontyphoidal Salmonella is a key target for a protective B1b cell antibody response. Proc Natl Acad Sci U S A 2009, 106:9803-9808.

41. Sambrook J: Russell DW: Molecular cloning: a laboratory manual. New York: CSHL Press; 2001.

42. Thompson JD, Gibson TJ, Higgins DG: Multiple sequence alignment using ClustalW and ClustalX. Current protocols in bioinformatics / editoral board, Andreas D Baxevanis [et al] 2002, Chapter 2:Unit 2-Unit 3.

43. Rossiter AE, Browning DF, Leyton DL, Johnson MD, Godfrey RE, Wardius CA, Desvaux M, Cunningham AF, Ruiz-Perez F, Nataro JP, et al: Transcription of the plasmid-encoded toxin gene from enteroaggregative Escherichia coli is regulated by a novel co-activation mechanism involving CRP and Fis. Mol Microbiol 2011, 81:179-191.

44. Al-Hasani K, Henderson IR, Sakellaris H, Rajakumar K, Grant T, Nataro JP, Robins-Browne R, Adler B: The sigA gene which is borne on the she pathogenicity island of Shigella flexneri 2a encodes an exported cytopathic protease involved in intestinal fluid accumulation. Infect Immun 2000, 68:2457-2463.

45. Parham NJ, Srinivasan U, Desvaux M, Foxman B, Marrs CF, Henderson IR: $\mathrm{PicU}$, a second serine protease autotransporter of uropathogenic Escherichia coli. FEMS Microbiol Lett 2004, 230:73-83.

46. Eslava C, Navarro-Garcia F, Czeczulin JR, Henderson IR, Cravioto A, Nataro JP: Pet, an autotransporter enterotoxin from enteroaggregative Escherichia coli. Infect Immun 1998, 66:3155-3163.

47. Hewitt CJ, Nebe-von Caron G, Nienow AW, McFarlane CM: The use of multi-parameter flow cytometry to compare the physiological response of Escherichia coli W3110 to glucose limitation during batch, fed-batch and continuous culture cultivations. J Biotechnol 1999, 75:251-264

48. Garen A, Levinthal C: A fine-structure genetic and chemical study of the enzyme alkaline phosphatase of $E$. coli. I. Purification and characterization of alkaline phosphatase. Biochim Biophys Acta 1960, 38:470-483.

49. Arnold K, Bordoli L, Kopp J, Schwede T: The SWISS-MODEL workspace: a web-based environment for protein structure homology modelling. Bioinformatics 2006, 22:195-201.

50. Kelley LA, Sternberg MJ: Protein structure prediction on the Web: a case study using the Phyre server. Nat Protoc 2009, 4:363-371.

51. Buchan DW, Ward SM, Lobley AE, Nugent TC, Bryson K, Jones DT: Protein annotation and modelling servers at University College London. Nucleic Acids Res 2010, 38:W563-W568.

doi:10.1186/1475-2859-11-69

Cite this article as: Sevastsyanovich et al:: A generalised module for the selective extracellular accumulation of recombinant proteins. Microbial Cell Factories 2012 11:69. 